\title{
Mini-clusters of potentially prodromal symptoms may identify psychiatrically well Amish children at higher risk of developing bipolar I disorder
}

Egeland JA, Shaw JA, Endicott J, et al. Prospective study of prodromal features for bipolarity in well Amish children. J Am Acad Child Adolesc Psychiatry 2003;42:786-96.

Do frequencies of potential early prodromal clinical features for bipolar I (BPI) disorder in psychiatrically well Amish children correlate with family history, and therefore inferred risk, of BPI?

METHODS

$\square$

Design: Prospective cohort study; outcome assessors were blinded as to family history, however, it was impossible to blind data collectors.

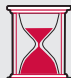

Follow up period: Seven years.

Setting: Amish families in rural Pennsylvania, USA; 1994 to 2000

을 People: 210 children from 27 families. 14 BPI families (100 children) had one parent with BPI. The families of same sex, psychiatrically unaffected siblings of each bipolar parent were selected as matched controls where possible. Control siblings were selected to be as close in age, marriage date, and number of children to the bipolar sibling. In the absence of a suitable matched sibling control, the family of a non-related Amish participant matched for sex, age, and family size, but without a family history of BPI, was selected. There were 9 sibling control families (77 children) and 4 family history negative control families (33 children).

Risk factors: The Child and Adolescent Research Evaluation (CARE) interview was administered annually to parents of participating children. The CARE interview comprised three parts: part $A$ (yes/no questions) pertained to the pregnancy/delivery, medical, and developmental history of the child; part B was narrative and asked parents whether they had any concerns about their child's health, wellbeing, or behaviour; part C (yes/ no questions) was used in alternate years and comprised 40 questions about the child being "noticeably different" from other children for potentially prodromal or antecedent items, and about the child's functioning and "wellness".

Outcomes: Presence of prodromal symptoms and predicted risk of developing BPI disorder as determined by independent, blinded assessors (one psychiatrist, two child psychiatrists, and one clinical psychologist).

\section{MAIN RESULTS}

Children with one BPI parent were significantly more likely to have 7 or more potentially prodromal symptom/behaviour items than children with well parents (AR: $12 \%$ for BPI family child $v 3 \%$ for control family child; $\mathrm{p}=0.0007)$. Symptoms significantly more

For correspondence Dr Egeland, University ö Miami North Research Office Briarcrest Square, Hershey, Pennsylvania, USA; OOAmish@aol.com

Sources of funding: The CARE (Child and Adolescent Research and Evaluation) programme was supported in part by the Stanley Medical Research Institute and with funding from the University of Miami School of Medicine. frequent in children with one BPI parent than in children with well parents were: anxiety, poor attention span in school, low energy, excitability, hyper-alertness, mood lability, school role impairment, sensitivity, somatic complaints, and stubbornness $(\mathrm{p} \leqslant 0.05)$. Assessors identified more of the children with one BPI parent as "at risk" of BPI than children with well parents (AR: $38 \%$ for BPI family child $v 17 \%$ for control family child; overall p value for liability class distribution $<0.003$ ).

\section{CONCLUSIONS}

Mini-clusters of early potential prodromal features occur at higher frequencies in psychiatrically well Amish children with one parent with BPI than in those with well parents.

\section{NOTES}

The authors note that the major limitation of this interim report of their study is that although these findings do identify potential early prodromal features for BPI, the true test of the outcome will be whether children designated as "at risk" do eventually develop BPI.

\section{Commentary}

Tis paper describes a prospective study of 210 healthy old-order Amish children, designed to determine the pattern of psychiatric symptoms that may be prodromal for bipolar disorder. Data are the result of 7 years of annual interviews with families begun in 1994 as part of the Child and Adolescent Research Evaluation (CARE) programme, designed for the above purpose. It is a novel study design producing useful data for several reasons.

While several studies have collected retrospective data from inpatients on selected prodromal bipolar symptoms or prospective data on such symptoms in children from affected families, none has used a community sample including children of varying levels of risk for bipolar disorder. Further, as they have studied an Amish group, they have been able to collect multigenerational data in large families useful for genetic studies, within a cultural community with much social stability. These group data on early bipolar risk may not be applicable to a general population, as symptoms of disruptive behaviour disorders-common in bipolar children-are possibly mitigated by cultural influences. However, this study has allowed the Egeland team to describe a spectrum of prodromal symptoms that may be specific for bipolar disorder and prove to be of relevance to clinical practice.

Of clinical importance, they found that children with bipolar parents have high risk of developing bipolar disorder $-38 \%$, similar to rates cited in other risk studies. Children of unaffected siblings of a bipolar adult had intermediate risk compared with control children, suggesting reduced penetrance of bipolar symptomatology in families. Symptoms of children of a bipolar parent, which are significantly more common than in children of non-bipolar parents, are described. It is suggested that some prodromal symptoms for bipolar disorder are episodic in $50 \%$ of the children, such as mood and energy changes, need for sleep, and anger/ temper outbursts. These findings will be helpful for clinicians assessing bipolar risk in children with subsyndromal symptoms combined with known or suspected family history of the illness.

Khrista Boylan, MD

Department of Psychiatry and Behavioural Neurosciences, McMaster University, Hamilton, Ontario, Canada 